\title{
MASSIVE DISTRIBUTED IRS AIDED WIRELESS COMMUNICATION WITH ON/OFF SELECTION
}

\author{
Riku Ohmiya ${ }^{1}$, Tomoki Murakami ${ }^{1}$, Masaaki Nishino², Yoghitha Ramamoorthi ${ }^{1}$, Masashi Iwabuchi' ${ }^{1}$, \\ Tomoaki Ogawa ${ }^{1}$ and Yasushi Takatori ${ }^{1}$ \\ ${ }^{1}$ NTT Access Network Service Systems Laboratories 1-1 Hikarinooka, Yokosuka-shi, Kanagawa, 239-0847 Japan, \\ ${ }^{2}$ NTT Communication Science Laboratories 2-4 Hikaridai, Seika-cho, Soraku-gun, Kyoto, 619-0237 Japan. \\ NOTE: Corresponding author: Riku Ohmiya, riku.oomiya.ez@hco.ntt.co.jp
}

\begin{abstract}
The mobile communication system beyond fifth-generation (beyond $5 G$ ) is required to maintain higher transmission capacity and reliability than existing 5G systems. To meet these requirements, one promising solution is to incorporate an Intelligent Reflecting Surface (IRS). The IRS involves reconfiguring the wireless propagation environment and exploiting the radio resources, not only in the conventional frequency and time domains but also in the spatial domain by controlling the reflection amplitude and phase of the incident electromagnetic signal. However, increased deployment of IRSs brings about interference and greater complexity. Therefore, in this paper, we propose an ON/OFF IRS selection method on massive IRS aided wireless communications that can simultaneously achieve interference suppression and capacity improvement. In addition, the proposed method reduces operational complexity using a simple 1-bit control. The results of computer simulation and experiment show that the proposed method can improve the desired channel capacity beyond directly Single-Input Single-Output (SISO) communication without IRS while maintaining the interference level of an interfered receiver below a certain threshold.
\end{abstract}

Keywords - Channel capacity, clustering methods, intelligent reflecting surface (IRS), interference, optimization

\section{INTRODUCTION}

Mobile communication traffic continues to increase due to the expanded use of smart devices such as smartphones and tablets. According to the International Telecommunication Union Radiocommunication Sector (ITU-R) [1], worldwide mobile traffic is increasing at an annual rate of 1.5 and is expected to exceed 5,000 exabytes (EB) per month by 2030. Expansion of the transmission capacity of today's mobile network is, therefore, an important issue going forward. As of February 2021, commercialization of the fifth-generation mobile communications system (5G) has begun in several countries, but discussions on the beyond fifthgeneration (beyond 5G), the new mobile network planned for 2030, are proceeding in parallel. Specifically, there is a need to achieve an ultra-hightransmission-capacity and ultra-high-reliability mobile network in beyond $5 \mathrm{G}$ at levels higher than that of 5G. This requirement is driving a transition to multifaceted research and development [2].

To meet the complex requirements of the radio layer in beyond $5 \mathrm{G}$, it is essential that radio resources can be exploited not only through technical innovations in the frequency and time domains as has been pursued up to now but also in the spatial domain. To utilize all the available radio resources, it is important to avoid Non Line-Of-Sight (NLOS) signals and constructively use multipath signals [2]. However, from the perspective of system operation, there are concerns that increasing the number of propagation paths would require the installation of a large number of base stations and antennas, which increases the cost of infrastructure.

Against this background, research and development have recently begun on new technology for controlling the propagation path itself using a reflecting device called an Intelligent Reflecting Surface (IRS) [3]. A reflector incorporating the IRS transforms into an active reflector having reflection characteristics different from those of an ordinary object. It can control the phase and amplitude of the incident radio-wave enable or disable reflection, control its direction, etc., without having to perform complex signal processing. If multiple distributed IRS reflectors can be deployed and their operation controlled in a coordinated manner, it should be possible to make maximum use of radio resources not only in the frequency and time domains as usual but also in the spatial domain.

A number of studies have been conducted on IRS reflector-aided wireless communication [3-9]. The study by Basar et al. [3] advances the concept of 
"smart radio environments" as a means of propagation path control by IRS reflectors. It also models the basic characteristics of IRS reflectors and clarifies the differences with other relay-system devices. Furthermore, as specific use cases, there are intelligent walls [4-6] and hyper-surfaces [7-9]. An intelligent wall is an IRS reflector that can enable/disable reflection and control phase $[0, \pi]$ with respect to the incident radio wave. It can be achieved using a PIN diode or variable resonator. Hyper-surfaces, meanwhile, centrally control IRS reflectors through a control unit equipped with an IoT gateway. In addition, a propagation path control is being theoretically formulated as a programmable wireless environment in which the maximizing of received signal intensity and the minimizing of maximum delay spread are objective functions.

Past studies have targeted a single, relatively large area IRS reflector, but to improve the performance of an entire wireless service area beyond indoor environments and certain small areas, there are also reports of obtaining even better effects by deploying and controlling distributed IRS reflectors at multiple locations [10][11]. There are also studies on maximizing the channel capacity of all single-antenna terminals by jointly optimizing the phase control of distributed IRS reflectors and basestation beam-forming control [12][13]. In order to flexibly meet various requirements such as higher transmission capacity and reliability in the beyond $5 \mathrm{G}$ era, it is necessary to expand to "massive" distributed IRS aided wireless communication as envisioned in this paper.

On the other hand, a distributed arrangement means a very large number of patterns in the way that the control parameters of each IRS reflector can be combined. Most of the previous literature assumes that the amplitude and phase parameters are continuously controlled for each element of the IRS reflectors [10-12]. However, the practical difficulty lies in the amount of time required for controlling and optimizing these and thus increases the operational complexity of IRS reflectors itself [14]. In addition, the effects of interference caused by an increase in the number of propagation paths must be considered in actual environments. To the best of our knowledge, this is the first work that performs combinatorial optimization of a large number of IRS reflectors while considering this interference.
Based on the above motivation, we propose a massive distributed IRS based on a 1-bit control IRS reflector with few elements in which all elements can be controlled for reflection (ON) or penetration (OFF) at the same time. Although 1-bit control is a rough control, the squared power gain is asymptotic to that of continuous control even for 1-bit controlled IRS in [14]. Instead of controlling the amplitude and phase parameters for each element of the IRS reflector, this paper proposes a formulation to select the ON/OFF combination of IRS reflectors. In particular, we formulate the joint ON/OFF IRS selection method and interference suppression as a non-linear binary integer programming problem. We then solve the formulated problem of IRS reflectors ON/OFF using the optimization solver while maintaining the interference level below a certain threshold. Further, a clustering method of IRS reflector is also proposed to reduce the computational complexity during the massive deployment of IRS. The proposed method performs clustering IRS reflectors having similar characteristics and selects only the representative value of a cluster as a combination candidate to keep computational costs constant even in the case of many IRS reflectors. We evaluate the effectiveness of the proposed method using simulation and experimental data.

The rest of this paper is organized as follows. Section 2 describes the system model used in this study and formulates the problem of suppressing interference by selecting IRS reflectors. Section 3 describes the method of clustering IRS reflectors proposed in this paper. Section 4 evaluates the performance of the proposed method by computer simulation and compares it with conventional clustering methods. Section 5 performs an evaluation using experimental data. Finally, Section 6 concludes the paper.

\section{MASSIVE DISTRIBUTED IRS AIDED WIRELESS COMMUNICATION WITH ON/OFF SELECTION}

\subsection{System model}

As shown in Fig. 1, this paper considers a system concept where Base Stations (BS) and User Equipment (UE) communicate via IRS reflectors. IRS reflectors are massively distributed, as they are installed on the roofs and walls of buildings. This environment is modeled as shown in Fig. 2. The same frequency is used here. This model consists of sender $S$, receiver $D$, interfered receiver $P$, and IRS 


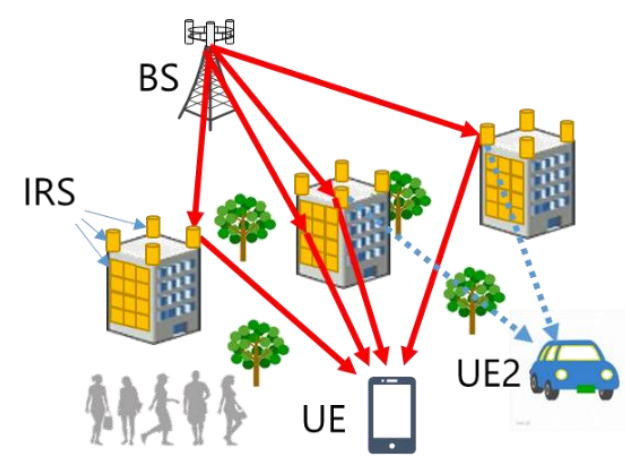

Fig. 1 -Massive distributed IRS aided communication

reflector $R$ with 1-bit control of reflect (ON) or penetrate (OFF). One way to implement this binary control is to incorporate PIN diodes into the metal parts of the IRS reflector and switch it with an external bias [4]. Also, the IRS reflector with few elements turns all the elements ON and OFF at the same time, like with or without a metal reflector. This assumption can greatly simplify the operational complexity of optimizing the states of the IRS reflective elements, making it an IRS combinatorial selection problem. Sender $S$ transmits signal $x$ toward receiver $D$ so as not to give interference to interfered receiver $P$. The signal of sender $S$ has the potential of becoming harmful interference to interfered receiver $P$. To solve this problem, combination $\boldsymbol{M}$ of IRS reflectors $R_{n}$ must be determined so as to suppress the interference on interfered receiver $P$ and maximize the channel capacity of sender $S$ and receiver $D$. Combination matrix $\boldsymbol{M}$ can be written as follows:

$$
\begin{aligned}
& \boldsymbol{M}=\left[\begin{array}{ccccc}
S_{R_{1}} & 0 & \cdots & \cdots & 0 \\
0 & \ddots & \ddots & \ddots & \vdots \\
\vdots & \ddots & S_{R_{n}} & \ddots & \vdots \\
\vdots & \ddots & \ddots & \ddots & 0 \\
0 & \cdots & \cdots & 0 & S_{R_{N}}
\end{array}\right], \\
& s_{R_{n}}=\left\{\begin{array}{cc}
1 & \text { selected } \\
0 & \text { not selected },
\end{array}\right.
\end{aligned}
$$

where $s_{R_{n}}$ is a binary variable that specifies whether the IRS reflector $R_{n}$ is selected (ON) or not (OFF).

The propagation channel from sender $S$ to interfered receiver $P$ via IRS reflectors $R_{n}$ can be expressed as the product of propagation channels $h_{S R_{n}}$ between sender $S$ and IRS reflectors $R_{n}$ and propagation channels $h_{R_{n} P}$ between IRS reflectors $R_{n}$ and interfered receiver $P$ as follows:

$$
h_{S R_{i} P}=h_{S R_{i}} h_{R_{i} P}=a_{P_{i}}+j b_{P_{i}}
$$

Similarly, the propagation channel from sender $S$ to receiver $D$ via IRS reflectors $R_{n}$ can be expressed as follows:

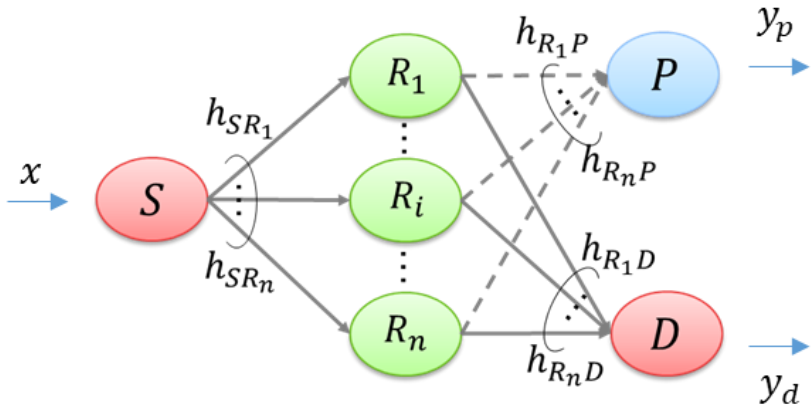

Fig. 2 -System model

$$
h_{S R_{i} D}=h_{S R_{i}} h_{R_{i} D}=c_{D_{i}}+j d_{D_{i}}
$$

Here, $a_{P_{i}}$ and $c_{D_{i}}$ are the real parts and $b_{P_{i}}$ and $d_{D_{i}}$ are the imaginary parts. $j$ is an imaginary unit.

When selecting distributed IRS reflectors, the propagation channel of interfered receiver $P$ can be expressed as the sum of the propagation channels via the selected IRS reflectors. The same can be said to hold for receiver $D$. Accordingly, interfered signal $y_{p}$ at interfered receiver $P$ and received signal $y_{d}$ at receiver $D$ can be written as follows:

$$
\begin{array}{r}
{\left[\begin{array}{l}
y_{p} \\
y_{d}
\end{array}\right]=\left[\begin{array}{l}
\boldsymbol{h}_{R P} \\
\boldsymbol{h}_{R D}
\end{array}\right] \boldsymbol{M h}_{S R} x+\left[\begin{array}{l}
n_{p} \\
n_{d}
\end{array}\right],} \\
\boldsymbol{h}_{R P}=\left[\begin{array}{lllll}
h_{R_{1} P} & \cdots & h_{R_{n} P} & \cdots & h_{R_{N} P}
\end{array}\right], \\
\boldsymbol{h}_{R D}=\left[\begin{array}{lllll}
h_{R_{1} D} & \cdots & h_{R_{n} D} & \cdots & h_{R_{N} D}
\end{array}\right], \\
\boldsymbol{h}_{S R}=\left[\begin{array}{lllll}
h_{S R_{1}} & \cdots & h_{S R_{n}} & \cdots & h_{S R_{N}}
\end{array}\right]^{T},
\end{array}
$$

where $n_{p}$ and $n_{d}$ represent the additive white Gaussian noise.

\subsection{Formulation of optimization problem}

The purpose of this subsection is to determine the IRS reflector combination $\boldsymbol{M}$ that can maximize the channel capacity of sender $S$ and receiver $D$ while maintaining the interference level of interfered receiver $P$ below a certain value.

The signal $x$ in Eq. (5) varies depending on the sum of the selected IRS channels. If we substitute the coefficient of signal $x$ with $h_{P}$ and $h_{D}$, we get:

$$
\begin{gathered}
{\left[\begin{array}{l}
y_{p} \\
y_{d}
\end{array}\right]=\left[\begin{array}{l}
h_{P} \\
h_{D}
\end{array}\right] x+\left[\begin{array}{l}
n_{p} \\
n_{d}
\end{array}\right],} \\
h_{P}=\left(\sum_{i=I}^{N} a_{P_{i}} s_{R_{i}}\right)+j\left(\sum_{i=I}^{N} b_{P_{i}} s_{R_{i}}\right), \\
h_{D}=\left(\sum_{i=I}^{N} c_{D_{i}} s_{R_{i}}\right)+j\left(\sum_{i=I}^{N} d_{D_{i}} s_{R_{i}}\right),
\end{gathered}
$$


Now, denoting the Signal-to-Noise Ratio (SNR) as $\gamma$, the channel capacity $C_{D}$ of sender $S$ and receiver $D$ can be written as:

$$
C_{D}=\log _{2}\left(1+\gamma h_{D}^{2}\right),
$$

In addition, the Interference-power-to-Noise Ratio (INR) of interfered receiver $P$ can be written as:

$$
\operatorname{INR}=2^{\log _{2}\left(1+h_{P}{ }^{2}\right)}-1=\gamma h_{P}{ }^{2},
$$

From the above, the optimization problem to be solved in this paper can be written as follows:

$$
\begin{gathered}
\max \left(\sum_{i=I}^{N} a_{D_{i}} s_{R_{i}}\right)^{2}+\left(\sum_{i=I}^{N} b_{D_{i}} s_{R_{i}}\right)^{2} \\
\text { s.t. }\left(\sum_{i=I}^{N} c_{P_{i}} s_{R_{i}}\right)^{2}+\left(\sum_{i=I}^{N} d_{P_{i}} s_{R_{i}}\right)^{2} \leq \frac{\mathrm{INR}}{\gamma} \\
S_{R_{i}} \in\{0,1\}
\end{gathered}
$$

Eq. (14) indicates the objective function by maximizing the channel capacity of sender $S$ and receiver $D$, Eq. (15) specifies to maintain the interference level of interfered receiver $P$ below the threshold $\left(\frac{\mathrm{INR}}{\gamma}\right)$ and Eq. (16) is a binary variable constraint and specifies whether the IRS reflector has been selected or not.

Here, variable $s_{R_{i}}$ is an integer that takes on the value of 0 or 1 while the objective function and limiting conditions are quadratic functions, so this problem constitutes a nonlinear binary integer programming problem. This type of problem is NPhard and is known to be a problem in which the computational time increases exponentially [15].

One example of a technique for solving a formulated optimization problem is a method that uses a dedicated program called a solver. In this paper, we use Solving Constraint Integer Programs (SCIP), which is relatively fast among general-purpose solvers and available for non-commercial applications [16]. Given the optimization problem, a general-purpose solver can output optimal values and an optimal solution at high speed based on that formula although the specific calculation method is a black box.

We compared the computational time between a method that performs a simple exhaustive search and a method that searches for a solution using the SCIP solver. The CPU of the computer used in this evaluation was an Intel Xeon W-2125 @ 4GHz. For the case of searching the combinations of 20 IRS reflectors (approximately 1,040,000 combinations), the exhaustive search required 9.5 seconds on average while SCIP was able to find a solution in 1 second on average. These results indicate that the computational time can be speeded up by approximately 10 times when using SCIP.

The SCIP solver can solve the nonlinear 0-1 integer programming problem that we would like to solve in this paper, but there is still the problem that the computational time increases exponentially as the number of IRS reflectors to be selected increases.

\section{IRS REFLECTOR CLUSTERING}

In this paper, we additionally propose a method for keeping computational complexity down while maintaining interference suppression performance by efficiently thinning out IRS reflectors as candidates in a combinatorial search using radio characteristics. Specifically, this method partitions a total of $N$ IRS reflectors into $K$ clusters according to a certain rule and selects from each cluster the IRS reflector closest to the center of gravity of that cluster as a candidate for the combinatorial search. In the following, we explain in detail the random method [17], k-means method [18], and proposed method for the sake of comparing different techniques.

\subsection{Random method}

This method selects $K$ IRS reflectors randomly from a total of $N$ IRS reflectors without clustering as candidates for a combinatorial search. This is an extremely simple and high-speed method, but as the value of $K$ increases, the probability of selecting duplicate IRS reflectors having similar characteristics likewise increases, which may result in a drop in interference suppression performance.

\section{2 k-means method}

This method clusters together IRS reflectors having similar propagation channel characteristics on an IQ complex plane and selects one representative IRS reflector from that cluster as a combinatorial search candidate. In this regard, the propagation channel from the sender to the receiver and interfered receiver via IRS reflectors can be expressed in the form of complex numbers as in Eq. (3) and Eq. (4). Here, we consider mapping the channel characteristics to the interfered receiver (Eq. (3)) onto an I-Q complex plane and forming clusters of IRS reflectors having close characteristics. 


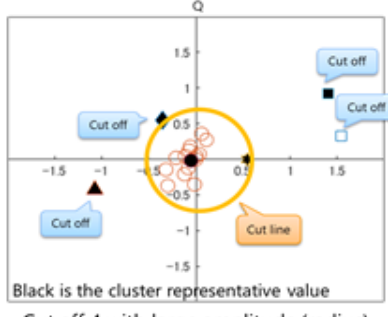

Cut off 4 with large amplitude (radius)

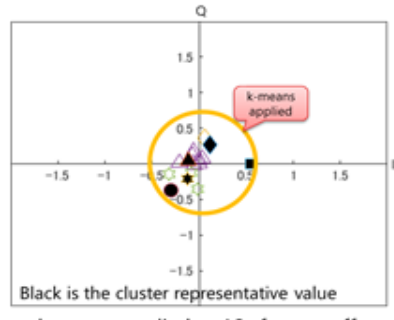

k-means applied to 16 after cut off
Fig. 3 - Clustering by proposed method

We formulate the k-means method [18] in this paper as follows. Denoting the center of gravity of each cluster as $\mu_{k}$ and that cluster as $M\left(\mu_{k}\right)$, we define variable $q_{i k}$ that indicates whether the IRS reflector with channel characteristics $h_{S R_{i} P}$ is included in cluster $k$ as follows:

$$
q_{i k}=\left\{\begin{array}{cc}
1 & h_{S R_{i} P} \in M\left(\mu_{k}\right) \\
0 & \text { otherwise }
\end{array}\right.
$$

The objective function to be minimized can be written as follows:

$$
J\left(q_{i k}, \mu_{k}\right)=\sum_{i=1}^{N} \sum_{k=1}^{K} q_{i k}\left\|h_{S R_{i} P}-\mu_{k}\right\|^{2},
$$

The results of partitioning the channel characteristics of a total of 20 IRS reflectors into 5 clusters using the k-means method are shown in Fig. 3 (left). The five symbols $\circ, \diamond, \triangle, \square$, and is each represent a reflector classified as belonging to one of those 5 clusters with a black symbol indicating the representative value of that cluster. The representative value of a cluster is set in the IRS reflector closest to that cluster's center of gravity.

However, applying the k-means method directly to the clustering of IRS reflectors is not suitable. If it is assumed that the propagation channel characteristics of an IRS reflector have a multipath configuration, the channel amplitude component, i.e., the radius on the I-Q plane, takes on the Rayleigh distribution while the phase component takes on a uniform distribution. In particular, for a small number of clusters, it is difficult to form a cluster of IRS reflectors with large amplitudes, and as a result, the five clusters become divided into two sections: one cluster with small amplitudes concentrated near the origin ( $O$ in Fig. 3 (left)) and multiple sparse clusters or "outliers" with amplitudes and phases that are greatly different $(\diamond, \triangle$, $\square$, and is in Fig. 3 (left)). Considering that the objective of this paper is to select a combination of IRS reflectors that can cancel out the interference level of an interfered receiver, the difference in amplitudes between clusters is kept as small as possible.
Table 1 - Simulation paramteters

\begin{tabular}{|c|c|}
\hline Parameter & Value \\
\hline \hline Number of senders & 1 \\
\hline Number of receivers & 1 \\
\hline Number of interfered receivers & 1 \\
\hline Number of IRS reflectors & 20,100 \\
\hline Number of antennas & 1 \\
\hline Propagation model & i.i.d. Rayleigh fading \\
\hline SNR & $10 \mathrm{~dB}$ \\
\hline Interference threshold (INR) & $1 \mathrm{~dB}$ \\
\hline Loops & 1000 \\
\hline
\end{tabular}

\subsection{Proposed method}

Taking the above into account, we considered that these "outlier" IRS reflectors could be "cut off" in the method proposed in the paper. However, since various propagation characteristics exist in the actual environment, the proposed method can improve the transmission capacity according to the propagation environment by controlling the threshold value of cut off. Specifically, denoting the total number of IRS reflectors as $N$ and the number of clusters as $K$, the method cuts off $N / K$ number of IRS reflectors with large amplitudes and applies the k-means method to the remaining $N-(N / K)$ IRS reflectors. In Fig. 3 (right), the four reflectors with large amplitudes are cut off from the 20 reflectors mapped in Fig. 3 (left) and the k-means method is applied to the remaining 16 reflectors. It can be seen here that the one cluster concentrated near the origin in Fig. 3 (left) has been broken down into the four clusters in Fig. 3 (right) and that differences in amplitude have been kept fixed.

The proposed method can therefore find a solution using a solver. The IRS reflectors here serve as a representative value of clusters after cutting off outlier IRS reflectors and applying the k-means method. This method is considered to be particularly effective for a small number of cluster partitions. This is because increasing the number of partitions causes its performance to asymptotically approach that of the standard k-means method.

\section{SIMULATION EVALUATION}

In this section, we evaluate the effectiveness of the proposed method by computer simulation using MATLAB. First, we describe the simulation environment. As listed in Table 1, we prepared one sender $S$, one receiver $D$, one interfered receiver $P$, and 100 IRS reflectors $R$. Other simulation conditions are listed in Table 1. Here, with the aim of clarifying the basic characteristics of the 
proposed method and its characteristics in a variety of environments, we assume a propagation channel model featuring identically distributed (i.i.d.) Rayleigh fading (uncorrelated paths). In short, we substitute values for the Rayleigh distribution and uniform distribution in the amplitude and phase components, respectively, of Eq. (3) and Eq. (4). Furthermore, we set the SNR of each receiver, i.e., $\gamma$ in Eq. (12) and Eq. (13), to $10 \mathrm{~dB}$ and interference threshold (INR) to $1 \mathrm{~dB}$.

Now, denoting SNR as $\gamma$, the channel capacity of directly SISO communications not using IRS reflectors can be written as follows:

$$
C_{\text {SISO }}=\log _{2}(1+\gamma) \text {, }
$$

Since $\gamma$ in this simulation is $10 \mathrm{~dB}$, a channel capacity exceeding approximately $3.5 \mathrm{bit} / \mathrm{s} / \mathrm{Hz}$ would demonstrate the effectiveness of propagation path control by IRS reflectors.

\subsection{Comparison of channel capacities}

We evaluated the Cumulative Distribution Function (CDF) of channel capacity $C_{D}$ obtained by receiver $D$ (see Eq. (12)) when partitioning a total of 100 IRS reflectors into 5 or 10 clusters and inputting the representative values of those clusters into the solver. Here, to clarify the characteristics of the proposed method, we compare its results with those of a method that selects IRS reflectors randomly (random method) and a method that applies k-means clustering with no cut-offs (kmeans method).

The results of selecting 5 IRS reflectors from out of 100 IRS reflectors are shown in Fig. 4. With the proposed method, approximately half of the channel capacity values were greater than 4 $\mathrm{bit} / \mathrm{s} / \mathrm{Hz}$ thereby exceeding the channel capacity of SISO communications while maintaining the allowed amount of interference. Since the amount of interference is constrained as in Eq. (15), it is always less than the interference threshold. On the other hand, the performance of the k-means method was worse than that of the random method with approximately half of the channel capacity values falling under $1 \mathrm{bit} / \mathrm{s} / \mathrm{Hz}$. We consider the reason for this to be that the number of combinations satisfying the allowed INR of interfered receiver $P$ was small since the difference in amplitudes among the clusters was large, which decreased the number of combinations that could improve the capacity of receiver $D$.

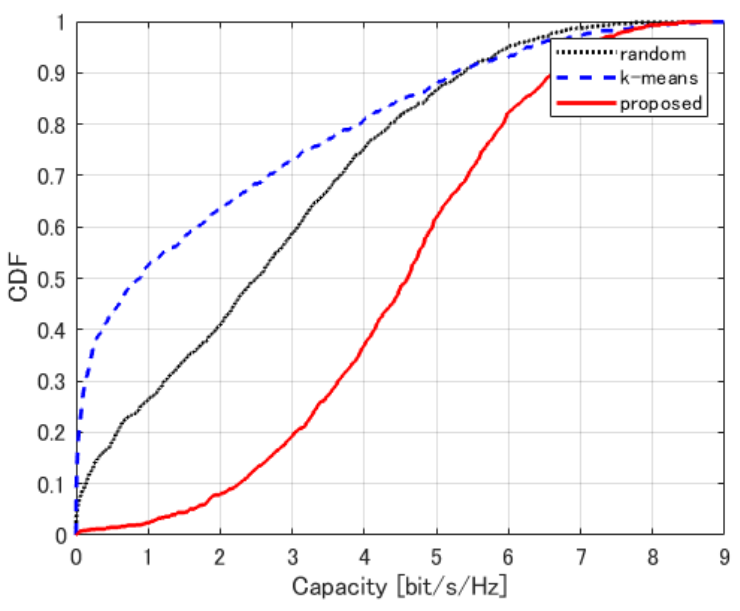

Fig. 4 - Channel capacity when partitioning into 5 clusters

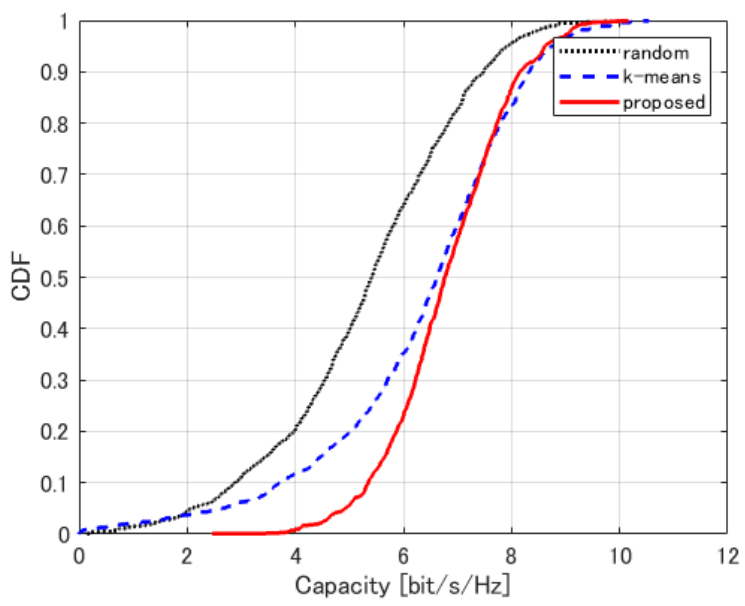

Fig. 5 - Channel capacity when partitioning into 10 clusters

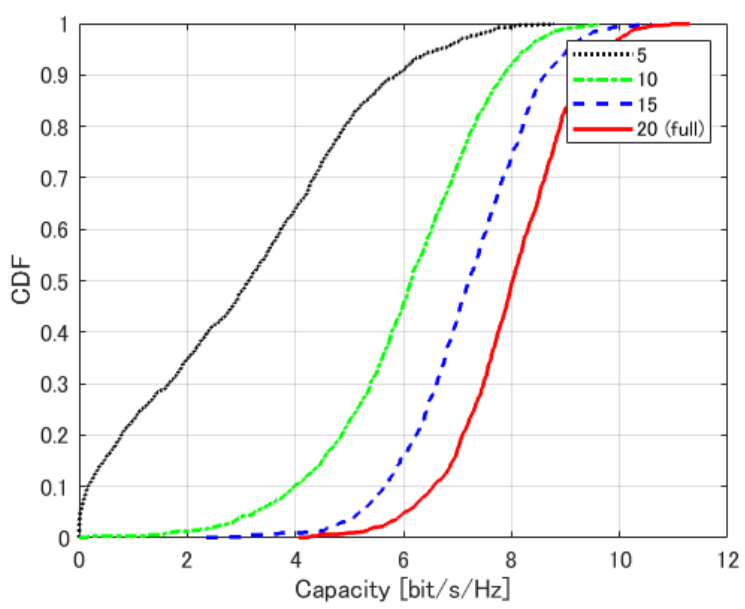

Fig. 6 - Number of cluster partitions versus channel capacity in the proposed method

The results of selecting 10 IRS reflectors from out of 100 IRS reflectors are shown in Fig. 5. For the random method and k-means method, the worst value was still $0 \mathrm{bit} / \mathrm{s} / \mathrm{Hz}$, but that of the proposed method was greatly improved to $2.5 \mathrm{bit} / \mathrm{s} / \mathrm{Hz}$ so that the channel capacity of SISO communications 


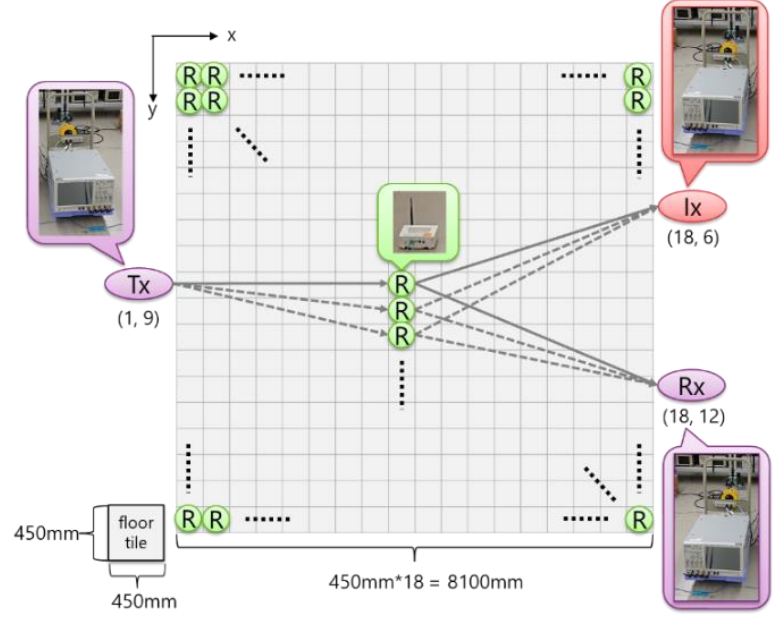

Fig. 7 - Experimental environment

was exceeded by nearly all of the combinations. However, as far as the best value is concerned, the proposed method asymptotically approached the kmeans method. The reason we give for this is that the distribution of amplitudes among the clusters could be kept fixed even for clustering by the kmeans method due to an increase in the number of cluster partitions.

\subsection{Number of clusters and performance}

Next, to clarify the relationship between the number of cluster partitions and channel capacity in the proposed method and to compare results obtained by the proposed method with those of an exhaustive search with no partitioning into clusters, we evaluated the channel capacity $C_{D}$ of receiver $D$ when partitioning a total of 20 IRS reflectors into 5 , 10,15 , and 20 (exhaustive search) clusters. Results are shown in Fig. 6. It can be seen that the performance of the proposed method increasingly deviated from that of the exhaustive search as the number of clusters decreased but that dividing the 20 IRS reflectors into half that number of clusters, i.e., 10 clusters, could secure a channel capacity of about $70 \%$ that of the exhaustive search.

\section{EXPERIMENTAL EVALUATION}

The characteristics of the proposed method depend greatly on the propagation channel, so evaluation based on actual channel measurements is essential for practical implementation. To therefore clarify its effectiveness in an actual environment, we measured an actual propagation channel using an oscilloscope and performed a simulation treating the measured values as a propagation channel passing through IRS reflectors.

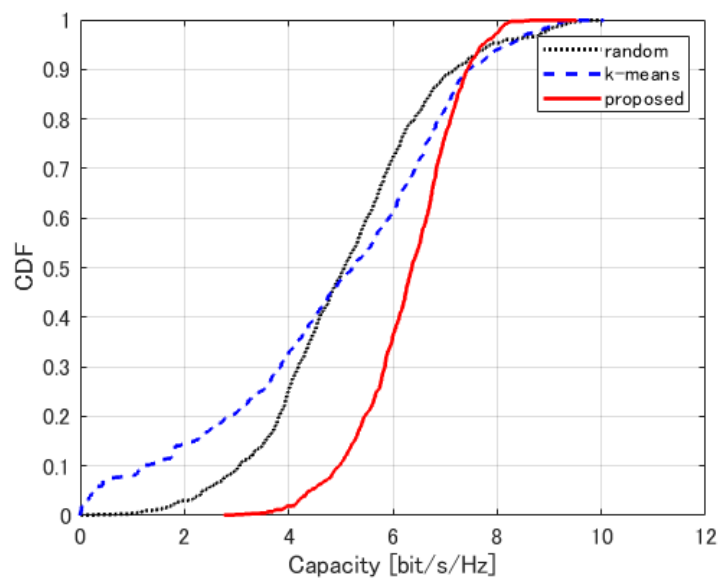

Fig. 8 - Results of 10-cluster partitioning based on experimental data

A conceptual diagram of the experiment is shown in Fig. 7. The experiment was conducted in a laboratory within the NTT Yokosuka R\&D Center. The floor of the laboratory was laid with $450-\mathrm{mm}$ square tiles and an $18 \times 18$ arrangement of these tiles were used in the experiment to secure an experimental space of 324 tiles in total. To indicate the positions of the experimental equipment, the upper left corner of the diagram in Fig. 7 was taken to be the origin with the $\mathrm{x}$-axis and $\mathrm{y}$-axis extending in rightward and downward directions, respectively. To apply the model shown in Fig. 2 to this space, we fixed the sender (Tx) at position $(1,9)$, the interfered receiver (Ix) at position $(18,6)$, and the receiver $(\mathrm{Rx})$ at position $(18,12)$ while enabling an IRS reflector $(\mathrm{R})$ to be set up at all positions from $(1,1)$ to $(18,18)$. In the experiment, a $5 \mathrm{GHz}$ signal generator (20 $\mathrm{MHz}$ bandwidth) specified by IEEE 802.11ac was set up on a tile and treated as an IRS reflector and the generated signal was measured by an oscilloscope (Keysight Technologies) set up at the positions of the sender, interfered receiver, and receiver. This operation was repeated to obtain the channel state information for all 324 locations where the IRS reflector was set up.

Now, on dividing these 324 channels obtained by experiment into 10 clusters and entering the representative value of each into the solver, we evaluated the CDF of channel capacity $C_{D}$ obtained by receiver $D$ (see Eq. (12)) the same as in subsection 4.2. Results are shown in Fig. 8. Similar to the results of Fig. 5 obtained by simulation, these experimental results also showed that the proposed method significantly increased the worst value for channel capacity from $0 \mathrm{bit} / \mathrm{s} / \mathrm{Hz}$ to $2.4 \mathrm{bit} / \mathrm{s} / \mathrm{Hz}$ compared with two conventional methods thereby demonstrating its effectiveness in improving propagation characteristics. 


\section{CONCLUSION}

In this paper, with the aim of improving the potential of wireless communications using massive distributed IRS reflectors in the coming beyond $5 \mathrm{G}$ era, we clarified by both simulation and experiment the effectiveness of the ON/OFF IRS reflectors selection method deployed in a distributed manner in an indoor LOS environment. We formulated this combination of IRS reflectors as an optimization problem of maximizing the receiver's channel capacity while minimizing interference on the interfered receiver and proposed a method for solving this problem at high speed using a general-purpose solver. We also proposed in combination with the above a method for extracting candidate IRS reflectors by clustering so as to decrease computational time while maintaining performance.

The results of the proposed method by a combination of IRS reflectors showed that desired channel capacity could be improved beyond that of SISO communication without IRS reflectors while maintaining the interference level on an interfered receiver below a certain threshold. It was also shown that the proposed clustering method could speed up processing by approximately 10 times compared with a method for finding a solution through an exhaustive search and that the worst value for the obtained channel capacity could be significantly improved from 0 to $2.4 \mathrm{bit} / \mathrm{s} / \mathrm{Hz}$. The proposed method was also effective for real channels acquired by experiments.

\section{REFERENCES}

[1] IMT traffic estimates for the years 2020 to 2030, Rep. M.2370-0, ITU-R, Jul. 2015.

[2] White Paper 5G Evolution and 6G, NTT DOCOMO, Jan. 2020.

[3] E. Basar, M. D. Renzo, J. D. Rosny, M. Debbah, M. Alouini, and R. Zhang, "Wireless Communications Through Reconfigurable Intelligent Surfaces," IEEE Access, vol. 7, pp. 116753-116773, 2019.

[4] L. Subrt and P. Pechac, "Controlling propagation environments using intelligent walls," in Proc. 2012 6th Eur. Conf. Antennas Propag. (EUCAP), pp. 1-5, Mar. 2012.
[5] T. J. Cui, M. Q. Qi, X. Wan, J. Zhao, and Q. Cheng, "Coding metamaterials digital metamaterials and programmable metamaterials," Light Sci. Appl., vol. 3, p. e218, Oct. 2014.

[6] S. V. Hum and J. Perruisseau-Carrier, "Reconfigurable reflectarrays and array lenses for dynamic antenna beam control: A review," IEEE Trans. Antennas Propag., vol. 62, no. 1, pp. 183-198, Jan. 2014.

[7] C. Liaskos, S. Nie, A. Tsioliaridou, A. Pitsillides, S. Ioannidis, and I. Akyildiz, "A novel communication paradigm for high capacity and security via programmable indoor wireless environments in next generation wireless systems," Ad Hoc Netw., vol. 87, pp. 1-16, May. 2019.

[8] C. Liaskos, S. Nie, A. Tsioliaridou, A. Pitsillides, S. Ioannidis, and I. Akyildiz, "Realizing wireless communication through softwaredefined hypersurface environments," in Proc. IEEE 19th Int. Symp. 'World Wireless Mobile Multimedia Netw.'(WoWMoM), pp. 14-15, Jun. 2018.

[9] C. Liaskos, S. Nie, A. Tsioliaridou, A. Pitsillides, S. Ioannidis, and I. Akyildiz, "A new wireless communication paradigm through softwarecontrolled metasurfaces," IEEE Commun. Mag., vol. 56, no. 9, pp. 162-169, Sep. 2018.

[10] M. Iwabuchi, T. Murakami, R. Ohmiya, T. Ogawa, Y. Takatori, Y. Kishiyama, and T. Asai, "[Invited] Intelligent Radio-Wave Design: Distributed Intelligent Reflecting Surface with Direction-based Control for Millimeterwave Communications," in 2020 Int. Conf. Emerging Tech. Commun. (ICETC), Dec. 2020.

[11] Y. Cao, T. Lv, and W. Ni, "Intelligent Reflecting Surface Aided Multi-User mmWave Communications for Coverage Enhancement," in 2020 IEEE 31st Int. Symp. Personal, Indoor and Mobile Radio Commun., pp. 1-6, 2020.

[12] Z. Li, M. Hua, Q. Wang, and Q. Song, "Weighted Sum-Rate Maximization for Multi-IRS Aided Cooperative Transmission," IEEE Wireless Commun. Letters, vol. 9, no. 10, pp.1620-1624, Oct. 2020. 
[13] P. Wang, J. Fang, X. Yuan, Z. Chen, and H. Li, "Intelligent Reflecting Surface-Assisted Millimeter Wave Communications: Joint Active and Passive Precoding Design," IEEE Trans. Vehicular Tech., vol. 69, no. 12, pp. 14960-14973, Dec. 2020.

[14] Q. Wu and R. Zhang, "Beamforming Optimization for Intelligent Reflecting Surface with Discrete Phase Shifts," in 2019 IEEE Int. Conf. on Acoustics, Speech and Signal Processing (ICASSP), 2019,

[15] X. Wang, W. Wu, and D. Zhu, "An Approximation Algorithm for Nonlinear 0-1 Integer Programming Problems," in Int. Conf. Computer and Management (CAMAN), pp. 1-5, 2011.

[16] "SCIP," [Online]. Available: https://scip.zib.de/.

[17] X. Meng, "Scalable simple random sampling and stratified sampling", Proc. of the 30th Int. Conf. on Machine Learning (ICML'13), vol. 28, pp. 531-539, Jun. 2013.

[18] J. MacQueen, "Some Methods for Classification and Analysis of Multivariate Observations", Proc. of the Fifth Berkeley Symp. on Math. Stat and Prob., vol. 1, pp. 281-296, 1967.

\section{AUTHORS}

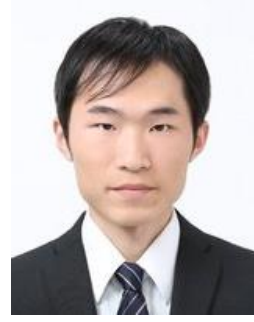

Riku Ohmiya received a B.S. degree in engineering and an M.E. degree in information science from Nagoya University, Nagoya, Japan, in 2016 and 2018, respectively. In 2018, he joined NTT Access Network Service Systems Laboratories, Yokosuka, Japan. His current research interests include smart radio environments for future wireless systems. He is a member of IEICE. He received the Young Researcher's Encouragement Award from IEICE IN of Japan in 2017 and Presentation Award for Young Researcher from IEICE RCS of Japan in 2019.

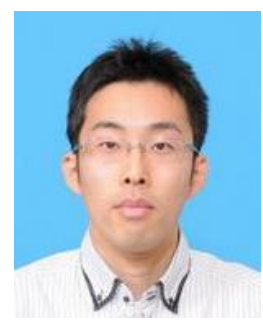

Tomoki Murakami received B.E., M.E., and Dr.Eng. degrees from Waseda University, Japan in 2006, 2008, and 2015, respectively. In 2008, he joined NTT Network innovation Laboratories, Nippon Telegraph and Telephone Corporation (NTT), Yokosuka, Japan. He is currently a research engineer in the Wireless Access Systems Project. His current research interests are high efficiency technologies for future wireless systems. He received the Young Engineer Award from the IEICE in 2010, the Active Research Award from IEICE AP (Antenna and Propagation) in 2010, the Best Tutorial Paper Award from IEICE Communications Society in 2014, Best Paper Award and the KIYASU-Zen'iti Award from the IEICE in 2015, and the Best Paper Award from IEICE on AP in 2016. He is a member of IEEE and IEICE.

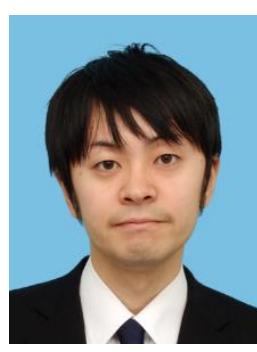

Masaaki Nishino received his B.E., M.E., and Ph.D. in informatics from Kyoto University, Japan, in 2006, 2008, and 2014, respectively. He joined NTT in 2008. His current research interests include combinatorial algorithms, combinatorial optimization, and natural language processing.

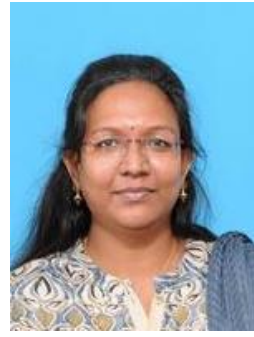

Yoghitha Ramamoorthi received B.E. and M.E. from Anna University, Chennai, India in 2012 and 2014, respectively. She received her Ph.D. from Department of Electrical Engineering, Indian Institute of Technology, Hyderabad in 2020. She is currently a Postdoctoral researcher in NTT Access Network Service System Laboratories, Yokosuka, Japan. Her research interests include energy efficiency, resource allocation, user scheduling, optimization of wireless networks, multiple access, and multi-connectivity technologies of next generation wireless networks. 


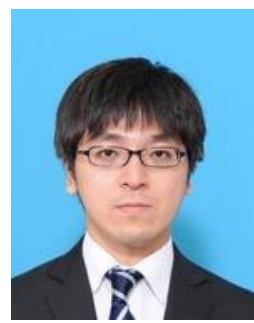

Masashi Iwabuchi received B.S. and M.S. degrees from the Tokyo Institute of Technology, Tokyo, Japan, in 2008 and 2010, respectively. From 2010 to 2016, he joined the NTT Access Network Service Systems Laboratories, Nippon Telegraph and Telephone Corporation (NTT). His current research interests are system design and radio access technologies for future radio access. He received the Young Engineers Award from the Institute of Electronics, Information and Communication Engineers (IEICE) in 2015. He is a member of the IEICE.

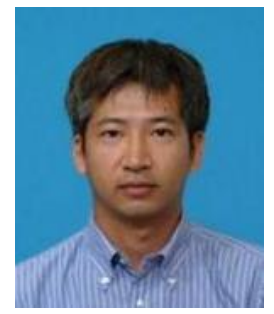

Tomoaki Ogawa received a B.A. in environmental information and an M.A. in media and governance from Keio University, Kanagawa, in 1996 and 1998. He joined NTT Network Innovation Laboratories in 1998, where he has been working on indoor-location systems. His recent interest focuses on the development of wireless local area network services. He is a member of IEICE.

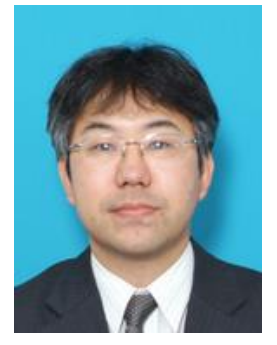

Yasushi Takatori Yasushi Takatori received B.E. and M.E. degrees from Tohoku University, Miyagi, Japan in 1993 and 1995, respectively. He received his Ph.D. degree in Wireless Communication Engineering from Aalborg University, Aalborg, Denmark in 2005. He joined NTT in 1995. He is currently working for NTT access network service systems laboratories as a senior distinguished researcher. He has served as a vice chairman of WLAN system development project in ARIB. His current research interests include future wireless access systems for Innovative Optical and Wireless Network (IOWN). He was a visiting researcher at the Center for TeleInFrastructure (CTIF), Aalborg University from 2004 to 2005 . He had served as a co-chair of COEX Adhoc in IEEE 802.11ac from 2009 to 2010 . He received the Best Paper Awards from IEICE in 2011, 2016 and 2020 respectively. He was honored with IEICE KIYASU Award in 2016. He received Radio Achievement Award from ARIB in 2020. He also received the IEEE Standards Association's Outstanding Contribution Appreciation Award for the development of IEEE 802.11ac-2013 in 2014. He is a senior member of IEICE and a member of IEEE. 